\title{
Left Ventricular Perfusion and Function at the Crossroads
}

\author{
Ami E Iskandrian, MD, MASNC ${ }^{a}$ \\ a Division of CV Diseases, Department of Medicine, University of Alabama at Birmingham, \\ Birmingham, AL
}

Received Aug 15, 2019; accepted Aug 15, 2019

doi: $10.1007 / \mathrm{s} 12350-019-01865-y$

It is well accepted that cardiac-gated positron emission tomography (PET) provides a plethora of information on myocardial blood flow (MBF), perfusion, and left ventricular function (LV) function. It is often less appreciated that gated single-photon emission computed tomography (G-SPECT) also provides a wealth of information in a single study. These include LV perfusion at rest or stress (or both), end-diastolic volume, end-systolic volume, ejection fraction (EF), mass, eccentricity index, systolic and diastolic synchrony (or lack of it), diastolic function, RV size, function and hypertrophy, and maybe, not too far in the future, on $\mathrm{MBF},{ }^{1-4}$ too. Many of these issues also apply to PET imaging.

The accuracy, precision, reproducibility, and repeatability of these indices will not be discussed here (I know some of our readers will be happy while others disappointed!) but what I would like to discuss is the correlation between LV perfusion and EF. In an ideal world, lack of perfusion defects (PD) should equate with normal EF and there would be a linear inverse relationship between PD size and EF. This may happen in some patients, but there are many exceptions, and the question remains: Why?

First, and before we go any further, I will remind you that $\mathrm{EF}$ is a time-honored measurement and continues to be so despite many new indices of systolic and diastolic LV function. Second, it is always important to double check the methods (EF and perfusion) when things do not add up: For example, normal-sized LV cavity, normal perfusion, but EF of $25 \%$, or large LV

\footnotetext{
Reprint requests: Ami E Iskandrian, MD, MASNC, Division of CV Diseases, Department of Medicine, University of Alabama at Birmingham, 316 LHRB/1900, UNIV BLVD, Birmingham, AL 35294; aiskand@uab.edu

J Nucl Cardiol 2020;27:1-2.

$1071-3581 / \$ 34.00$

Copyright (C) 2019 American Society of Nuclear Cardiology.
}

with a large defect and EF of $60 \%$. It does not, at first glance, make sense.

Here are my recommendations: (1) check the contours and be certain that the software is tracking the LV and not the gall bladder or some other extra cardiac activity, and that the visual assessment matched the computer-derived EF; (2) check the volume activity curve and determine if it is meaningful (end-systole is $\sim 1 / 3$ of RR cycle, and diastole has rapid filling phase, diastasis and atrial "kick" if heart rate is normal); (3) check ECG to determine if there are no obvious reasons for inappropriate tracking (low voltage, pacing spike, multiple premature beats, very irregular rhythm).

After that, check the perfusion part of the study, especially if there are apparent PD, and when automated programs are used to quantify the size. Be sure the contours are appropriate and consider that automated software, unlike human eyes, are vulnerable to the impact of motion artifacts or "hot spot" artifacts, just to mention a few.

In some patients, you may have only one set of data, a stress perfusion (if normal) and a post-stress EF. In others, you have 2 sets of data, stress perfusion and poststress $\mathrm{EF}$ and rest perfusion and post-rest EF. The issues with a rest study might be useful in considering the sources of variability between perfusion and function as it avoids, in most patients, the impact of ischemia-related variables. In rest studies, the perfusion is expressed either as rest summed score (RSS) or as \% of LV myocardium (regardless of the software program used).

1. When scoring PD, it is either normal, mildly decreased, moderately decreased, severely decreased, or absent, but there is no score for supernormalunlike wall motion (WM), where there is hyperkinesia (hyperdynamic).

2. The EF is load-dependent (preload, afterload, heart rate) and contractility-dependent. Patients may have concomitant diseases that affect EF but not perfusion 
such as aortic stenosis, cardiomyopathy, hypertension, or chronic kidney disease

3. Medications and interventions that affect EF such as inotropes, angiotensin converting enzyme inhibitors, beta-blockers, cardiac synchronization therapy

4. Remodeling where remote areas (outside areas with perfusion defects) become hypokinetic due to increased wall stress

5. Decreased resting myocardial blood flow due to repetitive stunning or hibernation

6. Incorrect interpretation, as some PD might be nothing more than attenuation artifacts

7. While we know any PD is abnormal, we do not know the baseline EF: For example, a drop from $75 \%$ to $50 \%$ represents a $33 \%$ decrease, and yet the $\mathrm{EF}$ is still normal!

When using post-stress EF and summed stress score (SSS) or \% total abnormality (fixed and reversible PD), post-stress stunning should also be considered.

Here are some key exit points to double check: (1) EF measurement whenever there is discordance between perfusion and function. At times repeat imaging or a rest study is needed, even though the stress perfusion is normal and a stress-only study was considered; (2) EF measurement when LV perfusion is normal but cavity is very dilated, except if dealing with aortic or mitral regurgitation, and (3) when there is a large PD but nearnormal EF. Please remember that the automated software will always provide EF value, but it is the user who has the responsibility to accept it.

Prior studies using rest and exercise radionuclide angiography showed that the prognostic value of rest
$\mathrm{EF} / \mathrm{LV}$-EF contains almost $80 \%$ of the prognostic value of exercise $\mathrm{EF}$ (or the change in EF from rest to exercise), which is why it is such a powerful measurement. The combined perfusion and function complement each other in predicting outcome since the perfusion pattern adds prognostic value to EF. ${ }^{1,2}$ Blind reliance on computer-generated numbers for either EF or perfusion at times is a disservice to our patients and to our field.

\section{Disclosure}

Ami E Iskandrian has nothing to disclose.

\section{References}

1. Iskandrian AE, Heo J, Mehta D, Tauxe EL, et al. Gated SPECT perfusion imaging for the simultaneous assessment of myocardial perfusion and ventricular function in the BARI2D trial: an initial report from the Nuclear Core Laboratory. J Nucl Cardiol 2006; 13:83-90

2. Iskandrian AE, Garcia EV, editors. Nuclear cardiac imaging principles and applications. (5th Edition). New York: Oxford University Press; 2015.

3. Iskandrian AE, Kegel JG, Tecce MA, et al. Simultaneous assessment of left ventricular perfusion and function with technetium99m sestamibi after coronary artery bypass grafting. Am Heart J 1993;126:1199-203.

4. Chen J, Henneman MM, Trimble MA. Bax JJ et al Assessment of left ventricular mechanical dyssynchrony by phase analysis of ECG-gated SPECT myocardial perfusion imaging. J Nucl Cardiol 2008; 15:127-36.

Publisher's Note Springer Nature remains neutral with regard to jurisdictional claims in published maps and institutional affiliations. 